\title{
NIVEL DE CONOCIMIENTOS DE PRIMEROS AUXILIOS EN ESTUDIANTES DE LA INSTITUCIÓN EDUCATIVA “JOSEFINA MEJÍA DE BOCANEGRA” NAZCA 2012.
}

Level of knowledge of first aid for students of School "Josefina Mejia Bocanegra" Nazca 2012

Gladys Leticia Muchaypiña Canales, ${ }^{1, a, b}$, Fanny Giovana Palomino Siviruero,

Erika Judith Peña Goy ${ }^{a}$

Policlínico Juan Pablo Elías S.C.R.L .Nasca, Ica, Peru.

${ }^{2}$ Facultad de Enfermería, Universidad Nacional San Luis Gonzaga de Ica. Ica, Peru.

${ }^{a}$ Lic. en Enfermería. ${ }^{b}$ Coordinadora de pruebas funcionales del Área de Salud Ocupacional del Policlinico Juan Pablo Elias S.C.R.L. Nasca, Ica, Perú.

\section{RESUMEN}

El presente estudio de investigación: Nivel de conocimientos de primeros auxilios en estudiantes de la Institución Educativa "Josefina Mejía de Bocanegra" Nazca 2012. Objetivo: Determinar el nivel de conocimientos en primeros auxilios en estudiantes de la Institución Educativa "Josefina Mejía de Bocanegra". Material y Métodos: El método que se utilizó fue el descriptivo y de corte transversal tomándose como muestra a 150 estudiantes del 5 to año de secundaria. La técnica que se utilizó fue la encuesta y como instrumentos se utilizaron formularios tipo cuestionarios de 23 ítems. Resultados: La participación de 150 estudiantes de quinto año de secundaria, la edad promedio fue 16,59 años, el 53,33\% de género femenino. El $62,00 \%$ habían recibido formación relacionado con primeros auxilios. El 86,6\% desaprobó el cuestionario, la nota promedio fue 6,9 puntos. Conclusiones: El nivel de conocimiento sobre primeros auxilios de los estudiantes de quinto año de secundaria evaluados es malo $(86,6 \%)$; Se sugiere evaluar y mejorar la formación práctica que brindan las Instituciones educativas en temas de primeros auxilios. Incluir los temas de primeros auxilios en la programación curricular.

Palabras Claves: Conocimientos, primeros auxilios, estudiantes.

\section{SUMMARY}

This research study: Level of knowledge of first aid students of School "Josefina Mejía Bocanegra" Nazca 2012. Objective: To determine the level of knowledge in first aid students of School "Josefina Mejía Bocanegra". Material and Methods: The method used was descriptive and cross-sectional sample taken as 150 students of 5th grade. The technique used was the survey forms and questionnaires as tools type 23 items were used. Results: The participation of 150 students from fifth year of high school, the average age was 16,59 years, $53,33 \%$ of the female gender. The $62,00 \%$ had received training related to first aid. $86,6 \%$ disapproved of the questionnaire, the average score was 6,9 points. Conclusions: The level of knowledge about first aid for students in the fifth year of secondary evaluated is bad (86,6\%); It is suggested to evaluate and improve the practical training offered by educational institutions in the areas of first aid. Topics include first aid in curricular programming.

Keywords: Knowledge, first aid, students. 


\section{INTRODUCCION}

Una vez que ocurren los accidentes, los primeros auxilios aplicados con rapidez son determinantes para reducir las secuelas y en ocasiones salvar vidas. De acuerdo a un informe de la OMS y el UNICEF, cada día mueren más de 2,000 niños debido a lesiones no intencionales (accidentales), y cada año ingresan decenas de millones con lesiones que a menudo los dejan discapacitados para toda la vida (1).

En Europa, Ellen Mason, miembro de la Fundación del Corazón, ha señalado que en una encuesta realizada a 2,000 ciudadanos demostró que sigue existiendo un gran desconocimiento sobre cómo actuar ante una muerte súbita (2). En España el RCP salva cada año el $20 \%$ de vidas de personas con paradas cardiacas (3). En México en el año de 2008, las estadísticas del Instituto Nacional de Estadística y Geografía (INEGI) registraron 20,152 fallecimientos por accidentes, los accidentes automovilísticos fueron los más comunes, seguidos por agresión, caídas, accidentes deportivos y lesiones autos infringidos.

En el Perú los accidentes de tránsito fatales se incrementan. Según el reciente informe técnico de Seguridad Ciudadana elaborado por el Instituto Nacional de Estadística e Informática (INEI), el 2011 se registraron, 2,794, 484 más que en el 2010, año en el que se registraron 2.310 (4).

Durante el 2010 se registró en Ica de 1,485 a $1,573(5,93 \%) ; \quad$ accidentes de tránsito encontrándose en el décimo departamento según el INEI de accidentes de tránsito en los departamentos del Perú (5).

La provincia de Nazca fue golpeada en 1996 por un violento terremoto de magnitud 6,4 en la escala de Richter, Según INDECI afecto a 6 localidades en 5 distritos, dejando 10 muertos ,1.132 heridos y 31339 damnificados (6).
Una comunidad sana puede alcanzar fácilmente sus metas de desarrollo, en tanto que es menos vulnerable (7). De allí la necesidad socioeducativa de crear una cultura, desde edades tempranas de la vida, para a largo plazo incidir en la disminución de la mortalidad pre hospitalaria por accidentes. La labor del personal de salud es fortalecer a las comunidades potenciando su autosuficiencia y perfeccionando sus actividades en materia de salud y primeros auxilios; y el ámbito escolar es el lugar indiscutible de la comunidad en la que el personal de salud debe organizarse para prevenir y atender las emergencias. Así pues directivos, docentes, personal no docente, y estudiantes pueden intervenir positivamente en la calidad de vida escolar. Los estudiantes de los últimos años de instituciones educativas, potencialmente, representan a la población que ha culminado todo un proceso de formación. Sin embargo, la educación para el manejo de primeros auxilios que se enseña en las instituciones parece ser inadecuada, en especial en los alumnos próximos a formar parte de la sociedad. Son pocas las instituciones educativas donde se imparte una enseñanza adecuada en estos temas, a pesar que en muchos colegios una problemática es la falta de cultura de prevención de desastres, Por lo que la institución educativa nacional "Josefina Mejía de Bocanegra" en la provincia de Nazca Ica, plantea en su PEI (proyecto educativo institucional) una solución dentro de los temas trasversales con una educación para la gestión de riesgos y conciencia ambiental.

En nuestro medio se ha encontrado poca literatura que aborde este problema.

El objetivo de este estudio fue Determinar el nivel de conocimientos de los estudiantes de la institución educativa en Nazca en primeros auxilios y la hipótesis planteada "EI Nivel de conocimientos de primeros auxilios en los estudiantes es malo". 


\section{MATERIAL Y METODOS}

El estudio de investigación fue de tipo cuantitativo y de nivel aplicativo, el método utilizado fue el descriptivo y de corte transversal,

La población estuvo conformada por 245 estudiantes del 5to año de secundaria de la Institución educativa "Josefina Mejía de Bocanegra" en Nazca, matriculados en el año 2012. Para la selección de la muestra, se utilizó la técnica de muestreo probabilístico sistemático aleatorio simple, la cual estuvo conformado por 150 estudiantes del 5to año. Se utilizó como instrumento el Cuestionario de Primeros Auxilios. Luego de la validación por los expertos, y siguiendo las sugerencias, se ha procedido a probar su confiabilidad el instrumento de recojo de información mediante el Alpha de Cronbach, aplicando a modo de test y retest $(0,875)$ en dos ocasiones en una muestra piloto de 10 estudiantes de $5^{\circ}$ año seleccionados al azar. Esta represento un buen nivel de confianza del instrumento utilizado.

\section{RESULTADOS}

Del 100\% (150 estudiantes) encuestados del 5to año de secundaria de la Institución educativa "Josefina Mejía de Bocanegra", tenemos lo siguiente: Según género, el mayor porcentaje de los encuestados son de género femenino. (Tabla № 1) La edad predominante es de 16 años $(54,7 \%)$, seguido por estudiantes con 17 años $(37,3 \%)$, en menor proporción están los estudiantes con 18 años (6,7\%), 15 años $(0,7 \%)$ y 20 años de edad (0,7\%). El promedio de edad de los estudiantes encuestados es de 16,53 años.

El $43,33 \%$ de los estudiantes recibieron enseñanza de primeros auxilios en forma superficial, $18,67 \%$ recibieron enseñanza teórica y práctica, $22,67 \%$ no recibieron enseñanza de primeros auxilios y el $15,33 \%$ no recuerdan haber recibido enseñanza sobre primeros auxilios. En conclusión $62 \%$ si recibieron enseñanza sobre primeros auxilios y $38 \%$ no.

El $36 \%$ de los estudiantes encuestados manifestaron que fue en el colegio actual donde recibieron la enseñanza sobre primeros auxilios, $15,33 \%$ afirmaron que fue en otras instituciones, $6 \%$ en otro colegio y $4,67 \%$ manifestaron que fue en una institución de salud donde recibieron la enseñanza sobre los primeros auxilios.

En cuanto a la antigüedad de los conocimientos en primeros auxilios , el $30,7 \%$ de los estudiantes afirman que fue hace no más de 12 meses que recibieron la enseñanza sobre primeros auxilios,, 12,7\% hace más de 5 años, $10 \%$ aseguran que pasaron más de 36 meses y $8,7 \%$ dicen que fue entre 12 a 36 meses.

El $86,6 \%$ de los estudiantes encuestados tienen un nivel malo de conocimiento sobre los primeros auxilios, el $11,3 \%$ tienen nivel regular, un $1,4 \%$ nivel bueno, y el $0,7 \%$ de los estudiantes tienen muy buen nivel. (TABLA № 2)

Según $x_{c}^{2} 2,57<3,84$, se afirma que no hay relación estadística significativa entre el Nivel de conocimiento sobre los primeros auxilios y el género. Sin embargo se aprecia una tendencia de que las mujeres en mayor proporción $(14=9,4 \%)$ conocen más sobre primeros auxilios respecto de los hombres ( 6 $=4 \%$ ). (TABLA № 3 )

$x_{c}^{2} 0,27<<5,99$, por lo tanto se afirma que no hay relación estadística significativa entre el nivel de conocimiento sobre los primeros auxilios y la edad.

Se aprecia una tendencia positiva de mayor conocimiento en el grupo etareo de 15 a 16 años de edad (11estudiantes) respecto a los de 17 años (7 estudiantes) y 18 a 20 (2 estudiantes). (TABLA № 4) 
TABLA № 1 : MUESTRA SEGÚN GÉNERO

\begin{tabular}{lcc}
\hline \multicolumn{1}{c}{ Género } & $\mathbf{N}^{\circ}$ & $\%$ \\
\hline Masculino & 70 & $46,7 \%$ \\
Femenino & 80 & $53,3 \%$ \\
Total & 150 & $100,0 \%$ \\
\hline
\end{tabular}

TABLA $N^{\circ}$ 2. CONOCIMIENTO SOBRE PRIMEROS AUXILIOS

\begin{tabular}{lcc}
\hline \multicolumn{1}{c}{ Conocimiento } & $\mathbf{N}^{\circ}$ & $\%$ \\
\hline Excelente: $16-17$ & 0 & 0 \\
Muy bueno: $14-15$ & 1 & 0,7 \\
Bueno: $12-13$ & 2 & 1,4 \\
Regular: $10-11$ & 17 & 11,3 \\
Malo $\leq 9$ & 130 & 86,6 \\
Total & 150 & $100,0 \%$ \\
\hline
\end{tabular}

TABLA N`3: RELACIÓN ENTRE EL NIVEL DE CONOCIMIENTO Y EL GÉNERO

\begin{tabular}{|c|c|c|c|c|c|}
\hline \multirow{2}{*}{$\begin{array}{c}\text { Nivel de } \\
\text { conocimiento }\end{array}$} & \multicolumn{2}{|c|}{ Género } & \multirow{2}{*}{ TOTAL } & \multirow{2}{*}{$\begin{array}{c}\mathrm{P}, 05 \% \\
95 \%\end{array}$} & \multirow[t]{2}{*}{$\alpha$} \\
\hline & Masculino & Femenino & & & \\
\hline$\geq$ Regular & 6 & 14 & 20 & $\mathrm{n}$ & 150 \\
\hline Malo & 64 & 66 & 130 & $x_{t}^{2} 3,84$ & \\
\hline Total & 70 & 80 & 150 & $\begin{array}{c}\mathrm{Gl} \\
x_{c}^{2} 2,57\end{array}$ & 1 \\
\hline
\end{tabular}

TABLA N4: RELACIÓN ENTRE EL NIVEL DE CONOCIMIENTO Y LA EDAD.

\begin{tabular}{|c|c|c|c|c|c|c|}
\hline \multirow{2}{*}{$\begin{array}{c}\text { Nivel de } \\
\text { conocimiento }\end{array}$} & \multicolumn{3}{|c|}{ Género } & \multirow{2}{*}{ TOTAL } & \multirow{2}{*}{$\begin{array}{l}\mathrm{P}, 05 \% \\
\text { n } 150\end{array}$} & \multirow{2}{*}{$\begin{array}{l}\alpha 95 \% \\
x_{t}^{2} \mathbf{5 , 9 9}\end{array}$} \\
\hline & $15-16$ & 17 & $18-20$ & & & \\
\hline$\geq$ Regular & 11 & 7 & 2 & 20 & gl 2 & $x_{c}^{2} 0,27$ \\
\hline Malo & 72 & 49 & 9 & 130 & & \\
\hline Total & 83 & & 56 & 150 & & \\
\hline
\end{tabular}

\section{DISCUSIÓN}

Nuestro objetivo fue determinar el nivel de conocimientos sobre primeros auxilios en los estudiantes de quinto año de secundaria. Basándonos en los resultados obtenidos encontramos que el $53,33 \%$ de los estudiantes encuestados son de sexo femenino, y $46,67 \%$ de sexo masculino. (TABLA $N^{\circ} 1$ ) $Y$ que el promedio de edad de los estudiantes encuestados es de 16,53 años. Un $62 \%$ de estudiantes manifestaron 
haber recibido enseñanza sobre primeros auxilios, de este porcentaje el $43,33 \%$ lo recibió de forma superficial, y el $18,67 \%$ recibieron enseñanza teórica y práctica, No obstante a ello se encontró como principal dato que el $86,6 \%$ de los estudiantes encuestados tuvieron resultados desaprobatorios, el promedio de calificación fue de 6,69 sobre un máximo de 17 puntos; El $86,6 \%$ de los estudiantes encuestados tienen un nivel malo de conocimiento sobre los primeros auxilios, $11,3 \%$ tienen nivel regular, $1,4 \%$ nivel bueno, y $0,7 \%$ de los estudiantes tienen muy buen nivel. Cabe resaltar que ninguno de los encuestados alcanzo el nivel de excelencia .Los estudiantes en un $71,3 \%$ conocen los signos universales de atragantamiento, más de la mitad sabe qué hacer ante un paro cardiaco, intoxicación y accidentes de tránsito. Por el contrario encontramos un desconocimiento marcado en identificar acciones a tomar en caso de hemorragias, convulsiones y desmayos. Del $62 \%$ de estudiantes que manifestaron haber recibido enseñanza sobre primeros auxilios, el $37,6 \%$ de los estudiantes encuestados no recuerdan el tiempo en el que recibieron la enseñanza sobre primeros auxilios, y un $30,1 \%$ afirman que fue durante el 2012 que recibieron dichos temas, el $12,9 \%$ manifiesta que fue hace más de 5 años es decir antes de comenzar la secundaria, el $10,8 \%$ aseguran que pasaron más de 36 meses y un porcentaje menor $8,6 \%$ dicen que fue entre 12 a 36 meses. En estudios similares sobre la Valoración de los primeros auxilios en estudiantes; muestras que estuvieron formadas por 358 alumnos, de los cuales $37,70 \%$ (135) son mujeres y el $62,30 \%$ (223) son hombres. Mostraron que el nivel de formación que poseen los estudiantes, en general, es muy bajo, tanto en los cursos analizados como en relación al sexo del alumno. De ahí que los resultados obtenidos sobre su conocimiento también sean preocupantes (8).
Según la revista cubana de salud pública. Comprobó que entre los escolares estudiados predomina el desconocimiento ante las situaciones de emergencia (9).

Estudios similares evidenciaron tendencias a obtener peores valores a medida que aumenta el tiempo en que se ha realizado el curso de formación (10).

Sin embargo, los resultados son contradictorios con la formación que dicen poseer los alumnos en primeros auxilios. Estos alumnos manifestaban haber recibido enseñanza en primeros auxilios, y obtienen unos resultados malos, similares a los alumnos que no recibieron formación. Según estudios de investigación, existe una marcada tendencia que a mayor nivel de conocimientos mejores serán las prácticas preventivas (11).

En un estudio titulado "Enseñanza de los primeros auxilios a escolares de 4to a 9no grados; en el que después de una intervención se obtuvo como resultados que el $60 \%$ de estudiantes tienen conocimientos en materia de primeros auxilios, y el $40 \%$ no tienen conocimientos (9).

Toda esta evidencia científica ha demostrado que la enseñanza de una adecuada práctica de Primeros Auxilios en los estudiantes, contribuye con el cambio en la disminución de la mortalidad por accidentes. Estos estudios se han evaluado en distintas poblaciones en instituciones de educación y en comunidades en general .Todas ellas apuntan que teniendo conocimientos básicos de primeros auxilios se consigue responder ante una situación de emergencia; y el modo de que éste resulte como algo habitual, necesario y de uso cotidiano, es introduciéndolo en la enseñanza obligatoria desde edades tempranas, adaptándolo a las posibilidades de desarrollo y aprendizaje de los alumnos. 


\section{CONCLUSIONES}

1. Se ha determinado que el nivel de conocimiento de los alumnos que cursan el quinto año de secundaria, respecto a los primeros auxilios es malo.

2. Más de la mitad de los alumnos de secundaria han recibido en algún momento formación en primeros auxilios; sin embargo estos cursos de formación son insuficientes puesto que ningún estudiante presenta formación de excelencia o muy buena. Llegando a la conclusión que los conocimientos son superficiales y no constituyen un aprendizaje significativo en el alumno.

\section{RECOMENDACIONES}

Se sugiere continuar fortaleciendo las investigaciones relacionadas al conocimiento de primeros auxilios y los factores demográficos y ambientales que influyen en ella, de la misma manera ampliar la muestra a todos los grados de estudios de secundaria. Además de evaluar y mejorar la formación práctica que brindan las Instituciones educativas en temas de primeros auxilios.

\section{Correspondencia:}

Gladys Leticia Muchaypiña Canales

Correo electrónico: soulmy17@hotmail.com

\section{REFERENCIAS BIBLIOGRAFICAS}

1. Peden M. Oyegbite K. Ozanne J. Hyder A. Branche C. Rahman A. Rivara F. Bartolomeos K. Cada dia mueren más de 2000 niños por lesiones no intencionales. (Sede web). WHO ; 2008 (Recuperado el 7 de febrero del 2011 ) Disponible en : http://www.who.int/mediacentre/news/rele ases/2008/pr46/es/

2. El Mundo "Salud - Corazón" Maniobras de Reanimación. Madrid, España 2012 [Consulta 15 de febrero del 2012]. Disponible en: http://www.elmundo.es/el mundosalud/2012/01/27/corazon/1327667 $\underline{771 . h t m l}$
3. Lesmes A. La reanimación cardiopulmonar. España, 2012. [Consulta 13 de junio del 2012] Disponible en: http://noticias.lainformacion.com/salud/enf ermedades-cardiacas/lareanimacioncardiopulmonar-evita-cada-ano-el-20-delas-muertes-depersonas-con-paradascardiacas QEdhBbAMe4n8bOa5T5iol6/

4. Ugarte C. Cada vez más peruanos mueren en accidentes de tránsito. Diario el Comercio. Miércoles 2 de mayo del 2012.

5. Documento Técnico: Anuario Estadístico 2010/ Ministerio del Interior. Dirección de Planeamiento Estratégico y Presupuesto. División de estadística - Lima Ministerio del Interior; 2011 (pág., 117)

6. Dirección de estadística- DNO-INDECI. Informe sobre el terremoto ocurrido en el sur del país el 12 de noviembre 96. Lima, Perú.2006 [consulta 9 julio del 2012]. Disponible en: http://www. indeci.gob.pe/ compend estad/1996/6 terre.pdf.

7. Federación Internacional de Sociedades de la Cruz Roja y de la Media Luna Roja. Guía de Implementación: Salud y primeros auxilios comunitarios (SPAC) en acción. Ginebra, Suiza. 2010 Disponible en http://www. ifrc.org/Global/Publications/Health/145 600 Implementation\%20guide SP LR.pdf

8. Abraldes, JA; Córcoles, CM; Muñoz, CM; Moreno, A. Valoración de los primeros auxilios en estudiantes de Educación Física. TRANCES. España. 2011. [Consulta 11 de junio del 2012]; Disponible en: http://www.trances.es/ papers /TCS\%2003 1 5.pdf 
9. Cazull I, Rodríguez A, Sanabria G., Hernández R. Enseñanza de los primeros auxilios a escolares de cuarto a noveno grados. Rev Cubana Salud Pública, Cuba. 2007 ; [Consulta 19 de Noviembre del 2012] Disponible en: http://scielo.sld.cu /scielo.php?script=sci arttext\&pid=S0864$34662007000200006 \& \operatorname{lng}=$ es \&nrm=iso

10. Abraldes J.A. y Ortín A. Conocimiento en Primeros Auxilios de los profesores de Educación Física en E.S.O. [tesis doctoral]. España: ; 2010
11. Colchado Y, Velasquez R. Nivel de conocimientos y prácticas preventivas sobre accidentes domésticos del cuidador del pre - escolar. Pueblo joven tres Estrellas - Chimbote 2006. [Tesis doctoral]. Lima: 2006 